\title{
Gallium Ga 68-NOTA-Aca-BBN(7-14)
}

National Cancer Institute

\section{Source}

National Cancer Institute. Gallium Ga 68-NOTA-Aca-BBN(7-14). NCI Thesaurus. Code C123929.

A radioconjug ate containing the bombesin (BBN) fragment $\mathrm{BBN}(7-14)$ comprised of the amino acid sequence of Gln-Trp-Ala-Val-Gly-His-Leu-Met-NH2, which targets the gastrin-releasing peptide receptor (GRPR), bound to aminocaproic acid (Aca), and linked by the macrocyclic chelating agent, 1,4,7-triazacyclononane- $\mathrm{N}, \mathrm{N}^{\prime}, \mathrm{N}^{\prime}$--triacetic acid (NOTA), to the radionuclide gallium (Ga) 68 , with potential use in diagnostic imaging using positron emission tomog raphy/computed tomog raphy (PET/CT). Upon administration of gallium Ga 68-NOTA-Aca-BBN(7-14), the peptide moiety targets and binds to GRPR. Upon PET/CT, GRPR-expressing tumor cells can then be visualized. GRPR, also called bombesin receptor 2 (BB2), is a seven-transmembrane G protein-coupled receptor belonging to the bombesin receptor family. It is overexpressed in certain types of cancers. 